Journal for ImmunoTherapy of Cancer

\section{Efficacy and safety of camrelizumab combined with apatinib in advanced triple-negative breast cancer: an open- label phase II trial}

To cite: Liu J, Liu Q, Li Y, et al. Efficacy and safety of camrelizumab combined with apatinib in advanced triplenegative breast cancer: an open-label phase II trial. Journal for ImmunoTherapy of Cancer 2020;8:e000696. doi:10.1136/ jitc-2020-000696

- Additional material is published online only. To view please visit the journal online (http://dx.doi.org/10.1136/jitc2020-000696).

$\mathrm{JL}$ and QL contributed equally.

Accepted 17 April 2020

Check for updates

(c) Author(s) (or their employer(s)) 2020. Re-use permitted under CC BY-NC. No commercial re-use. See rights and permissions. Published by BMJ.

For numbered affiliations see end of article.

\section{Correspondence to}

Dr Erwei Song;

songew@mail.sysu.edu.cn

Dr Qiang Liu;

victorlq@hotmail.com

Professor Zefei Jiang; jiangzefei@csco.org.cn

\section{ABSTRACT}

Background Previous trials showed that antiangiogenesis or anti-programmed death protein 1/programmed death ligand 1 (PD-1/PD-L1) monotherapy only showed marginal effect in triple-negative breast cancer (TNBC). Preclinical studies demonstrated that antiangiogenic therapy could sensitize breast cancer to PD-1/PD-L1 blockade via reprogramming tumor microenvironment. Combinational treatment of checkpoint blockade and antiangiogenesis for TNBC has not been reported.

Methods Patients with advanced TNBC with less than three lines of systemic therapy were enrolled in an openlabel, non-comparative, two-arm, phase II trial at Sun Yatsen Memorial Hospital. Camrelizumab (intravenously every 2 weeks) with apatinib orally at either continuous dosing (d1-d14) or intermittent dosing (d1-d7) was given until disease progression or unacceptable toxicities. Primary endpoint was objective response rate (ORR).

Results From January 2018 to April 2019, 40 patients were enrolled, including 10 in the apatinib intermittent dosing cohort and 30 in the apatinib continuous dosing cohort. The ORR was $43.3 \%$ (13 of 30 ) in the continuous dosing cohort, while no objective response was observed in the intermittent dosing cohort. The disease control rate was $63.3 \%$ (19 of 30) in the apatinib continuous dosing cohort, and $40.0 \%$ (4 of 10) in the apatinib intermittent dosing cohort, respectively. The median progressionfree survival (PFS) was $3.7(95 \% \mathrm{Cl} 2.0$ to 6.4$)$ months and $1.9(95 \% \mathrm{Cl} 1.8$ to 3.7$)$ months in the continuous dosing and intermittent dosing cohort, respectively. In the continuous dosing cohort, the median PFS of patients with partial response ( 8.3 months, $95 \% \mathrm{Cl} 5.9$ to not reached) was significantly longer than that of patients with stable disease/progressive disease/not evaluable (2.0 months, $95 \% \mathrm{Cl} 1.7$ to 3.0). The most common adverse events (AEs) included elevated aspartate aminotransferase/ alanine aminotransferase and hand-foot syndrome. Overall, $26.7 \%$ and $20.0 \%$ of patients experienced grade $\geq 3$ AEs in the continuous dosing and intermittent dosing cohort, respectively. In the continuous dosing cohort, a high percentage of baseline tumor-infiltrating lymphocytes $(>10 \%)$ was associated with higher ORR and favorable PFS ( $p=0.029,0.054$, respectively).

Conclusions The ORR by this chemo-free regimen was dramatically higher than previously reported ORR by anti-PD-1/PD-L1 antibody or apatinib monotherapy. Camrelizumab combined with apatinib demonstrated favorable therapeutic effects and a manageable safety profile in patients with advanced TNBC.

Trial registration number NCT03394287.

\section{INTRODUCTION}

Triple-negative breast cancer (TNBC) has a poor prognosis due to its aggressive features and lack of druggable targets. ${ }^{1}$ The median overall survival (OS) of metastatic TNBC is only 8-15 months. ${ }^{23}$ Chemotherapy remains the main systemic treatment for advanced TNBC, but drug resistance occurs rapidly and patients' tolerance is very poor. Therefore, there is a pressing need to develop novel therapeutic strategies for these patients.

Blockade of programmed death protein 1 (PD-1) and programmed death ligand 1 (PD-L1) emerges as an attractive therapeutic option for TNBC because stromal tumorinfiltrating lymphocytes (TILs) and PD-L1 are correlated with favorable outcomes in TNBC. ${ }^{4-7}$ However, monotherapy of PD-1/ PD-L1 blockade in advanced TNBC resulted in limited objective response rates (ORRs), ranging from $5.2 \%$ to $18.5 \% .^{8-10}$ These findings indicate the necessity of exploring combinational strategies with other treatments, including chemotherapy, radiotherapy or targeted therapies, to enhance the efficacy of checkpoint inhibitors. Recently, the IMpassion130 trial demonstrated that first-line treatment of atezolizumab (anti-PD-L1 antibody) with nab-paclitaxel led to a 2.2-month increase in progression-free survival (PFS) and a 7-month increase in OS than placebo plus nab-paclitaxel in patients with PD-L1positive advanced TNBC. ${ }^{11}$ Therefore, combinational strategy with immunotherapy is working. However, the optimal combinational 
approach has yet to come, especially for the patients with PD-L1-negative tumors or those who have received a few lines of chemotherapy.

Antiangiogenesis treatment was once thought to be promising in treating patients with TNBC, with bevacizumab approved by the Food and Drug Administration (FDA) in 2008 because of significantly increased PFS when combined with chemotherapy. Nevertheless, bevacizumab was removed from FDA approval in 2011 because it did not show OS benefit and had safety issues. Preclinical studies demonstrated that antiangiogenic therapies could sensitize anti-PD-1/PD-L1 treatment by increasing PD-L1 expression and $\mathrm{CD}^{+} \mathrm{T}$ cell infiltration in tumor microenvironment. ${ }^{12} 13$ Our preclinical study also found that low dose of antiangiogenic therapies sensitized breast carcinomas to PD-1 blockade via increasing the tumor infiltrating $\mathrm{CD} 8^{+} \mathrm{T}$ cells and $\mathrm{B}$ cells, and elevation of PD- 1 expression on $\mathrm{CD} 45^{+}$immune cells in tumor microenvironment. ${ }^{14}$ Thus, antiangiogenic therapies may enhance the response to PD-1/PD-L1 blockade and improve survival. Apatinib, an orally administered vascular endothelial growth factor receptor 2 (VEGFR2) tyrosine kinase inhibitor, has demonstrated antitumoral activity in several solid tumors ${ }^{15}$ although the ORR of apatinib monotherapy for patients with metastatic TNBC remained low in a multicenter trial. ${ }^{16}$ Camrelizumab (SHR-1210) is a fully humanized immunoglobulin G4/k PD-1 monoclonal antibody, which is well tolerated with positive antitumor activity in several kinds of solid tumors. ${ }^{17-19}$ Furthermore, the safety of camrelizumab combined with apatinib was explored in a phase I trial of patients with advanced hepatocellular carcinoma and gastric cancer, which demonstrated that this combination was well tolerated ${ }^{20}$ Here, we report the results of a phase II trial of camrelizumab in combination with apatinib in patients with advanced TNBC.

\section{METHODS}

\section{Study design and patients}

This was an open-label, randomized, parallel, noncomparative, two-arm, phase II trial. The key eligibility criteria were as follows: female patients at the age of 18-70 years with metastatic or unresectable recurrent TNBC (ER/PR-negative was defined as an ER/PR stain of $<1 \%$, HER2/Neu-negative was defined as immunohistochemistry (IHC) $0-1+$; and HER2/Neu-negative by chromogenic/fluorescent in situ hybridization, according to the American Society of Clinical Oncology (ASCO) guidelines); with measurable disease per Response Evaluation Criteria in Solid Tumors (RECIST) V.1.1; an Eastern Cooperative Oncology Group performance status of $0 / 1$; received $<3$ lines of systemic therapies for advanced/metastatic diseases; retained adequate organ and bone marrow function; and at least one obtainable representative metastatic or recurrent tumor sample. The exclusion criteria included: any history of autoimmune disease; metastasis to central nervous system with clinical symptoms; any active infection or recent treatment with a systemic immunostimulatory agent (within the previous 4 weeks); recent application of systemic glucocorticoid or immunosuppressive medication. Additionally, patients with a history of using antiangiogenesis agents or checkpoint inhibitors, or a history of severe allergic reaction to other monoclonal antibodies, or with uncontrollable hypertension or antihypertensive medication, or heart condition/disease were ineligible.

\section{Procedures}

Patients were randomly (1:1) enrolled to receive camrelizumab $200 \mathrm{mg}(3 \mathrm{mg} / \mathrm{kg}$ for patients whose weight was below $50 \mathrm{~kg}$ ) intravenously, once every two weeks (Q2W), in combination with apatinib $250 \mathrm{mg}$ oral, continuous dosing (d1-d14), or intermittent dosing (d1-d7) at the first stage of this trial. Since no patient achieved response in the apatinib intermittent dosing cohort at the first stage, we only enrolled patients into the apatinib continuous dosing cohort at the second stage. Initially, patients received up to 2 years of treatment. A subsequent protocol amendment allowed for treatment beyond 2 years until unacceptable toxicities or withdrawal of consent or disease progression per RECIST V.1.1. The dosage of camrelizumab was based on a prior phase I study. ${ }^{18}$ Also, the dosage of apatinib was according to the toxicity data from a phase I trial using camrelizumab plus apatinib. ${ }^{20}$

Tumor samples from metastases or recurrent tumors were acquired to confirm diagnosis of TNBC and to determine the expression of PD-L1 or other biomarkers on immune cells (ICs) or tumor cells (TCs). Therapeutic efficacy was assessed 8 weeks for the first 24 weeks and once every 12 weeks thereafter. Patients with progressive disease were determined by radiologic scans, as per RECIST V.1.1. The patients who were judged by investigators to have either potential pseudo progression, or were clinically benefiting, remained on treatment until they were confirmed to be disease progression by a second radiologic scan 4 weeks later. Adverse events (AEs) were graded according to the CTCAE, V.4.0.

Notably, apatinib was discontinued if a delay of $>4$ weeks was required for treatment-related AEs (TRAE). Camrelizumab was discontinued for any grade 3 or worse AEs until the toxicity resolved to grade 1 or less. Also, camrelizumab cannot be restarted if discontinued for more than 8 weeks. Furthermore, dose modification of camrelizumab was not allowed, while prespecified modifications of apatinib were permitted.

\section{Outcomes}

The primary end point was ORR. Secondary end points included disease control rate (DCR), duration of response (DOR), clinical benefit rate (CBR), time to response (TTR), PFS, 1-year OS rate, and toxicities.

For biomarker analysis, PD-L1 expression was measured using the FDA-cleared 22C3 assay on the Dako Link 48 platform. The positive threshold of PD-L1 on ICs/TCs was set at $\geq 1 \%$ PD-L1-expressing immune/tumor cells 
as percentage of tumor area. Stromal TILs were evaluated following the criteria proposed by the International TIL Working Group. ${ }^{21}$ CD8 immunofluorescence and PD-1 IHC assay were performed using anti-human CD8 (Cat. No. MA5-14548, Thermo Fisher; 1:200) and antihuman PD-1 (Cat. No. ab52587, Abcam; 1:50) antibody, respectively.

\section{Statistical analysis}

The two arms were randomized, non-comparative cohorts, and patient enrollment followed a Simon two-stage design. ${ }^{22}$ The design was used to test whether camrelizumab combined with apatinib yields an ORR that is of clinical interest. However, it limits the expected number of patients who receive treatment when the true ORR was not of clinical value. The two-stage test was conducted independently for each arm.

For each arm, the Simon design required 10 patients for the first stage and called for termination at stage 1 if there were less than two responders (complete response, CR or partial response, PR) among 10 patients. Otherwise, if two or more responders were identified in up to 10 patients, additional 19 patients would be enrolled. Treatment was considered of clinical interest if, at the end of the second stage, there were more than five responders among 29 patients in any single arm. With 29 patients in each arm, the trial had approximately $80 \%$ power to detect a true ORR of $\geq 30 \%$ against the true response rate of $10 \%$ with a two-sided type I error rate of $5 \%$.

DOR, TTR, PFS, and OS were evaluated by the KaplanMeier method. Log-rank test and $\chi^{2}$ test were used to assess associations between biomarkers and outcomes. Statistical analyses were performed using STATA (V.12.0). All statistical tests were two-sided, with statistical significance defined as $\mathrm{p}<0.05$.

\section{RESULTS}

\section{Patients and treatment}

From January 2018 to April 2019, 40 patients were enrolled at Sun Yat-sen Memorial Hospital, including 10 in the apatinib intermittent dosing cohort, and 30 in the apatinib continuous dosing cohort (figure 1). All patients $(n=40)$ were evaluable for safety, and 37 for overall response. On the data cut-off date on December 31, 2019, the median follow-up durations in the apatinib intermittent and continuous dosing cohort were 9.5 (range 2.7-20.0) months and 8.0 (range 1.7-23.0) months, respectively. Overall, $37(92.5 \%)$ had stage IV disease and $32(80.0 \%)$ had visceral metastases. Among them, 30 $(75.0 \%)$ had received at least one line of prior chemotherapy for advanced TNBC. Baseline characteristics were well balanced between the two cohorts (table 1).

On database lock, the median durations of treatment were 2.4 (range 0.9-5.6) months and 3.7 (range 0.5-21.7) months in the apatinib intermittent and continuous dosing cohort, respectively.

\section{Therapeutic efficacy}

The ORR in this study is shown in table 1 . Changes in tumor burden are shown in figure $2 \mathrm{~A}, \mathrm{~B}$, and disease burden over time in figure 2 . In the apatinib continuous dosing cohort, five patients had an overall response of PR at the first stage (evaluable $\mathrm{n}=10$ ). As a consequence, 20 patients were enrolled to this cohort at the second stage, with 18 being evaluable for ORR. Overall, the ORR was $43.3 \%(13 / 30,95 \%$ CI $25.5 \%$ to $62.6 \%)$ in the apatinib continuous dosing cohort. In contrast, no confirmed objective response was found at the first stage in the intermittent dosing cohort $(\mathrm{n}=10$, ORR $0 \%, 95 \%$ CI $0 \%$ to $30.8 \%$ ). Therefore, no further enrollment was taken in this cohort following the first stage. In the apatinib continuous dosing cohort, patients who received combination therapy as first-line therapy showed an ORR of $50.0 \%(5 / 10,95 \%$ CI $18.7 \%$ to $81.3 \%)$, while those who received it as second-line or third-line therapy had an ORR of $40.0 \%(8 / 20,95 \%$ CI $19.1 \%$ to $63.9 \%)$. The DCR was $63.3 \%(19 / 30,95 \%$ CI $43.9 \%$ to $80.1 \%)$ in the apatinib continuous dosing cohort and $40.0 \%$ (4/10, $95 \%$ CI $12.2 \%$ to $73.8 \%$ ) in the apatinib intermittent dosing cohort (table 2). Additionally, the CBR was $33.3 \%$ $(10 / 30,95 \%$ CI $17.3 \%$ to $52.8 \%)$ in the continuous dosing cohort and $10.0 \%(1 / 10,95 \%$ CI $0.3 \%$ to $44.5 \%)$ in the intermittent dosing cohort (table 2).

In the continuous dosing cohort, the median DOR and TTR were 6.6 (range 2.0-17.8) months (figure 3) and 1.9 (range 1.8-5.8) months, respectively. On analysis, $18 / 30(60.0 \%)$ and $8 / 10(80.0 \%)$ patients had died, and $21 / 30(70.0 \%)$ and $10 / 10(100.0 \%)$ had disease progression in the continuous and intermittent dosing cohorts, respectively. The median PFS was 3.7 (95\% CI 2.0 to 6.4 ) months in the continuous dosing cohort but 1.9 (95\% CI 1.8 to 3.7) months in the intermittent dosing cohort (figure 4A). The median OS was 8.1 (95\% CI 4.0 to not reached) months in the continuous dosing cohort and 9.5 (95\% CI 2.7 to 14.8) months in the intermittent dosing cohort. The 1-year OS rates were $42.2 \%$ (95\% CI 24.2 to 59.2 ) and $40.0 \%$ (95\% CI 12.3 to 67.0 ) for the continuous and intermittent dosing cohorts, respectively (figure 4B). Importantly, the median PFS of patients with an overall response of PR in the continuous dosing cohort was significantly improved compared with patients with stable disease $(\mathrm{SD}) /$ progressive disease $(\mathrm{PD}) /$ not evaluable (NE) $(8.3,95 \%$ CI 5.9 to not reached vs 2.0 , 95\% CI 1.7 to 3.0 months; $\mathrm{p}<0.0001$, online supplementary figure 1A). The 1-year OS rate of patients with an overall response of PR in the continuous dosing cohort was also significantly higher than those with $\mathrm{SD} / \mathrm{PD} / \mathrm{NE}$ (74.6\%, $95 \%$ CI 39.8 to 91.1 vs $17.7 \%$, $95 \%$ CI 4.4 to 38.3 , $\mathrm{p}=0.0003$, online supplementary figure $1 \mathrm{~B}$ ).

\section{Safety}

TRAE of any grade occurred in $9(90.0 \%)$ of 10 patients in the intermittent dosing cohort and in $30(100.0 \%)$ of 30 patients in the other cohort. Profiles of TRAE were similar in the two cohorts (table 3), which were manageable 


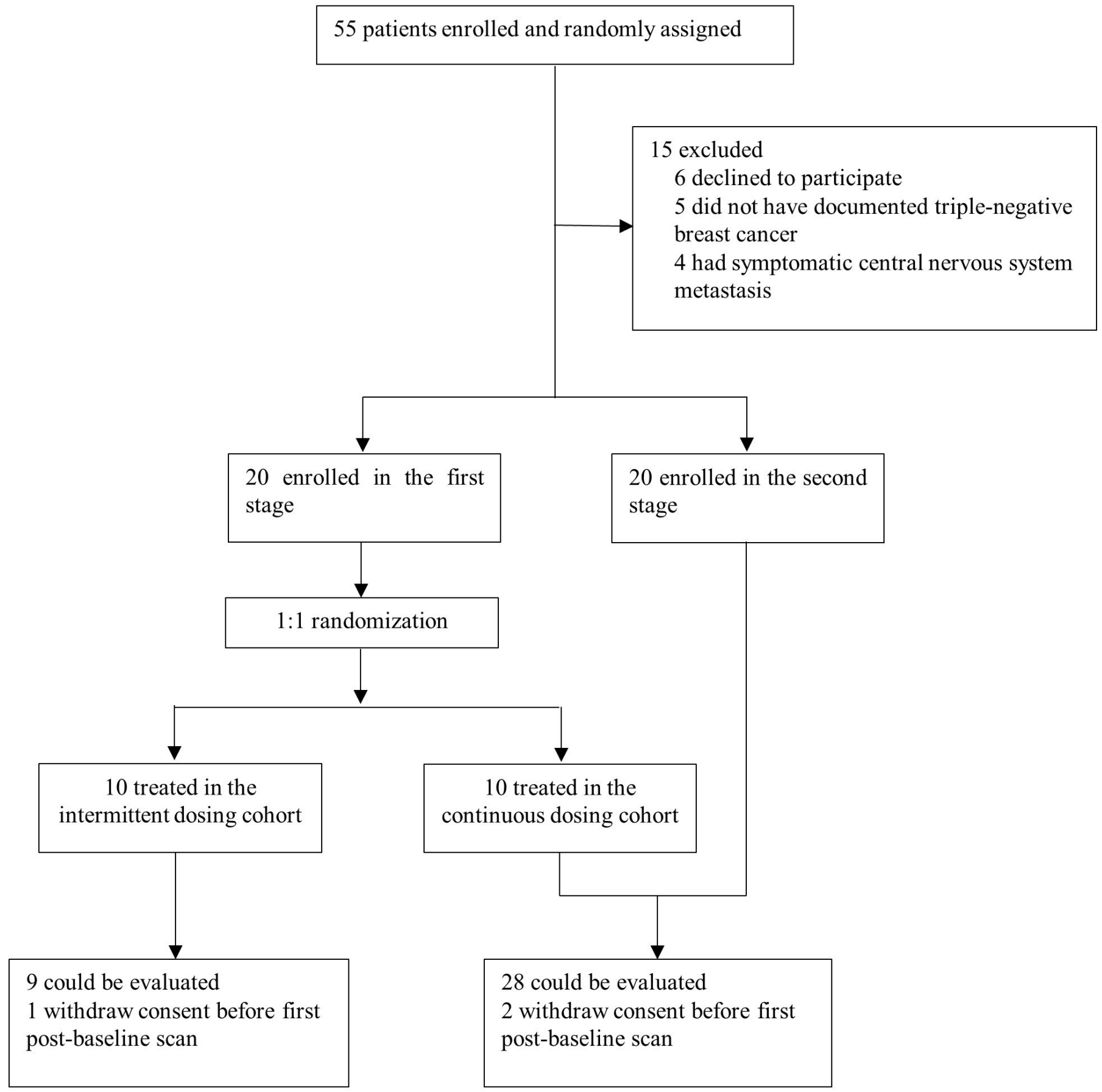

Figure 1 Trial profile.

with two patients in the continuous dosing cohort who discontinued treatment due to a grade 3 pneumonitis or a grade 3 proteinuria, respectively. The most common AE was elevated aspartate aminotransferase (AST) in both groups, but the majority were grade 1 or 2 elevations. Additionally, the incidences of hand-foot syndrome and hypertension were at least $6.0 \%$ higher in the continuous dosing group than in the intermittent dosing group. Moreover, two $(20.0 \%)$ patients suffered from grade 3 or 4 TRAE in the intermittent dosing group, while eight $(26.7 \%)$ experienced grade $\geq 3 \mathrm{AE}$ in the continuous dosing group (table 3). There were no treatment-related deaths.

The TRAEs of any grade classified as immune-related occurred in $7(70.0 \%)$ of 10 patients in the intermittent dosing group and $24(80.0 \%)$ of 30 patients in the continuous dosing group (online supplementary table 1 ). Among them, grade 3 or 4 immune-related AEs occurred in two $(20.0 \%)$ and five $(16.7 \%)$ patients in the intermittent and continuous dosing cohort, respectively (online supplementary table 1). Also, more patients in the continuous dosing cohort experienced immune-related 
Table 1 Baseline patient characteristics

\begin{tabular}{|c|c|c|c|}
\hline & $\begin{array}{l}\text { Continuous } \\
\text { dosing } \\
\text { cohort }(n=30)\end{array}$ & $\begin{array}{l}\text { Intermittent } \\
\text { dosing } \\
\text { cohort }(n=10)\end{array}$ & $\begin{array}{l}\text { All patients } \\
(n=40)\end{array}$ \\
\hline $\begin{array}{l}\text { Age, years, } \\
\text { median } \\
\text { (range) }\end{array}$ & $46.5(32-64)$ & $39.5(29-60)$ & $45.5(29-64)$ \\
\hline \multicolumn{4}{|c|}{ Prior therapy in advanced setting } \\
\hline No & $8(26.7)$ & $2(20.0)$ & $10(25.0)$ \\
\hline One line & $16(53.3)$ & $4(40.0)$ & $20(50.0)$ \\
\hline Two lines & $6(20.0)$ & $4(40.0)$ & $10(25.0)$ \\
\hline $\begin{array}{l}\text { Time on } \\
\text { prior therapy } \\
\text { before } \\
\text { enrollment, } \\
\text { months, } \\
\text { median } \\
\text { (range) }\end{array}$ & $4.9(0-29.3)$ & $5.1(0-20.5)$ & $5.1(0-29.3)$ \\
\hline $\begin{array}{l}\text { Metastatic } \\
\text { disease }\end{array}$ & $28(93.3)$ & $9(90.0)$ & 37 (92.5) \\
\hline
\end{tabular}

No. of sites of metastatic disease

\begin{tabular}{|c|c|c|c|}
\hline $0-3$ & $23(76.7)$ & $7(70.0)$ & $30(75.0)$ \\
\hline $\begin{array}{l}\geq 4 \text { site of } \\
\text { metastatic } \\
\text { disease }\end{array}$ & 7 (23.3) & $3(30.0)$ & $10(25.0)$ \\
\hline Bone & $6(20.0)$ & $4(40.0)$ & $10(25.0)$ \\
\hline Live & $8(26.7)$ & $4(40.0)$ & $12(30.0)$ \\
\hline Lung & $13(43.3)$ & $6(60.0)$ & $19(47.5)$ \\
\hline $\begin{array}{l}\text { Lymph } \\
\text { node only }\end{array}$ & $2(6.7)$ & $1(10.0)$ & $3(7.5)$ \\
\hline \multicolumn{4}{|c|}{ PD-L1 IC status } \\
\hline Negative & $18(60.0)$ & $8(80.0)$ & $26(65.0)$ \\
\hline Positive & $12(40.0)$ & $2(20.0)$ & $14(35.0)$ \\
\hline \multicolumn{4}{|c|}{ PD-L1 TC status } \\
\hline Negative & 19 (63.3) & $8(80.0)$ & $27(67.5)$ \\
\hline Positive & $11(36.7)$ & $2(20.0)$ & $13(32.5)$ \\
\hline
\end{tabular}

Data presented as $\mathrm{n}(\%)$ or median (range).

*Some patients had multiple sites of metastatic disease. IC, immune cells; TC, tumor cells.

hypothyroidism or hyperthyroidism than in the other cohort (26.7\% vs $10.0 \%$, and $16.7 \%$ vs 0 , respectively). Moreover, six patients in the continuous dosing cohort $(20.0 \%)$ required dose reduction of apatinib due to grade $3 \mathrm{AEs}$, while no patient in the intermittent dosing cohort had any AEs resulting in dose reduction or discontinuation.

\section{Biomarker analyses}

Previous studies have shown that a pre-existing immune tumor microenvironment, such as PD-L1 expression on TC or IC, stromal TILs and CD8 ${ }^{+} \mathrm{T}$ cells, and PD-1 expression on immune cells were associated with efficacy of antiPD-1/PD-L1 therapy. ${ }^{8112324}$ Therefore, we determined whether such a baseline immunological status was associated with clinical benefit from the combined therapy via exploratory biomarker analysis. Interestingly, neither PD-L1 expression on ICs nor that on TCs correlated with ORR ( $\mathrm{p}=0.569$ and 0.378 , respectively). PD-L1 IC or TC status also did not predict the benefits of PFS $(\mathrm{p}=0.717$ and 0.338 , respectively).

In this study, the cut-offs were $10 \%$ for stromal TILs, $0.5 \%$ for $\mathrm{CD}^{+} \mathrm{T}$ cells, and $1 \%$ for PD- 1 expression on ICs. In the continuous dosing cohort, a higher ORR and favorable PFS were observed in patients with a higher baseline TIL expression level $(\mathrm{p}=0.029,0.054$, respectively, online supplementary figures 2 and 3 ), but not in those with a higher infiltration of $\mathrm{CD}^{+} \mathrm{T}$ cells or higher PD-1 expression on ICs ( $\mathrm{p}=0.278,0.490$, and $0.657,0.784$, respectively).

\section{DISCUSSION}

In this phase II trial, we reported the efficacy, safety and potential predictive biomarkers for PD-1 inhibition with camrelizumab, plus apatinib that targets VEGFR2, for patients with advanced TNBC. To the best of our knowledge, this is the first trial that reports the efficacy and safety of combinational treatment of checkpoint inhibition and antiangiogenesis in TNBC. Our results showed that camrelizumab with apatinib was well tolerated in advanced TNBC, and demonstrated favorable ORR and PFS, regardless of lines of therapy and PD-L1 status. The ORR (43.3\%) achieved in this chemo-free regimen was dramatically higher than those in anti-PD-1/PD-L1 antibody $(5.2 \%-18.5 \%)$ or apatinib $(10.7 \%)$ monotherapy, ${ }^{8-10} 16$ demonstrating strong synergistic effects between these treatments.

Monotherapy of checkpoint inhibitors achieved response only in a small percentage of patients with TNBC, but patients could have significant survival benefit once they had a response. Thus, it is important to improve the response rate of anti-PD-1/PD-L1 therapies by combinational approaches. ${ }^{8-10}$ IMpassion 130 trial reported that first-line atezolizumab plus nab-paclitaxel led to significantly improved PFS than placebo plus nab-paclitaxel in advanced TNBC. However, only PD-L1-positive patients achieved OS benefit. ${ }^{11}$ Our current findings suggest that combinational therapy with anti-PD-1 antibody and VEGFR2 inhibitor significantly improved the ORR to 43.3\%. More importantly, even for patients with PD-L1negative tumors or patients received one to two lines of systemic therapies in the advanced setting, this novel combinational approach still showed favorable efficacy. This can be explained by the increased infiltration and enhanced activation of $\mathrm{CD}^{+} \mathrm{T}$ cells in tumor microenvironment, as well as by the PD-L1 upregulation in tumor, which are induced by antiangiogenic therapies. ${ }^{12} 13$ Also, our prior preclinical study demonstrated that VEGFR2 inhibitors including apatinib could normalize the tumor vasculature, and then increase the infiltration of antitumoral $\mathrm{CD}^{+} \mathrm{T}$ cells and $\mathrm{B}$ lymphocytes in breast 
The continuous dosing cohort

The intermittent dosing cohort
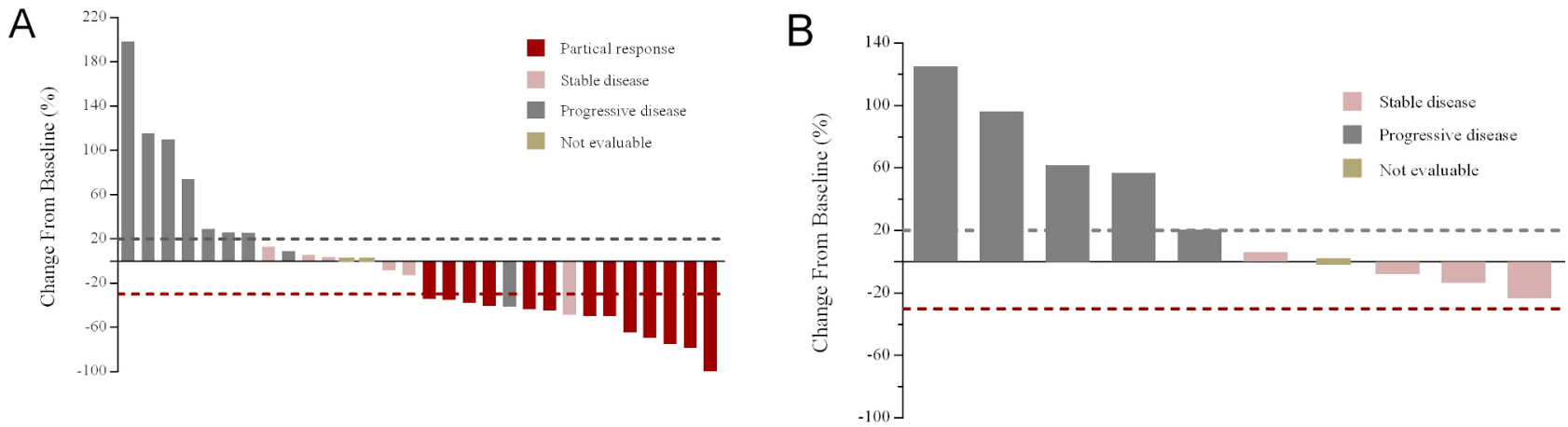

Best Overall Response

The continuous dosing cohort

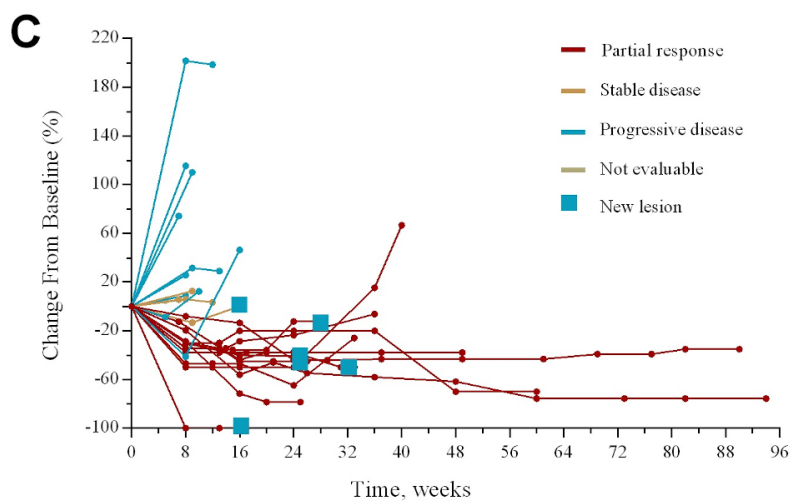

The intermittent dosing cohort

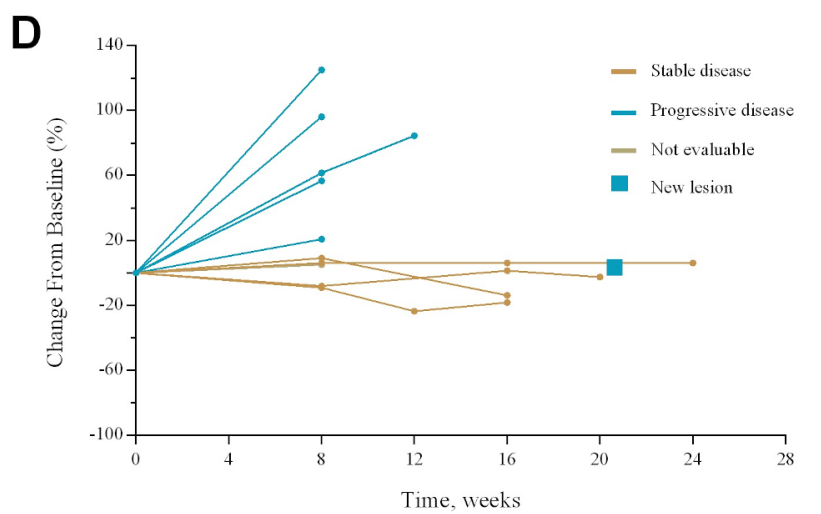

Disease Burden Over Time

Figure 2 Changes in tumor burden from baseline of the response-evaluable patients in the apatinib continues dosing cohort (A) and the apatinib intermittent dosing cohort (B). Disease burden over time in all enrolled patients in the apatinib continues dosing cohort (C) and the apatinib intermittent dosing cohort (D).

Table 2 Confirmed best overall responses

\begin{tabular}{|c|c|c|c|}
\hline & Continuous dosing cohort $(n=30)$ & Intermittent dosing cohort $(n=10)$ & All patients $(n=40)$ \\
\hline Objective response* & $13(43.3 ; 25.5-62.6)$ & $0(0 ; 0-30.8)$ & $13(32.5 ; 18.6-49.1)$ \\
\hline Disease control† & 19 (63.3; 43.9-80.1) & $4(40.0 ; 12.2-73.8)$ & $23(57.5 ; 40.9-73.0)$ \\
\hline \multicolumn{4}{|l|}{ Best overall response } \\
\hline Complete response & 0 & 0 & 0 \\
\hline Stable disease & $6(20.0)$ & $4(40.0)$ & $10(25.0)$ \\
\hline Progressive disease & $9(30.0)$ & $5(50.0)$ & $14(35.0)$ \\
\hline Not evaluable§ & $2(6.7)$ & $1(10.0)$ & $3(7.5)$ \\
\hline
\end{tabular}

Data are $\mathrm{n}(\% ; 95 \% \mathrm{Cl})$ or $\mathrm{n}(\%)$.

${ }^{*}$ Objective responses were confirmed by repeat imaging 4 weeks later.

†Includes patients who achieved an objective response or had stable disease as their best response.

flncludes patients who achieved an objective response or had stable disease as their best response for 24 weeks or more.

$\S$ Signifies patients with unevaluable responses. 


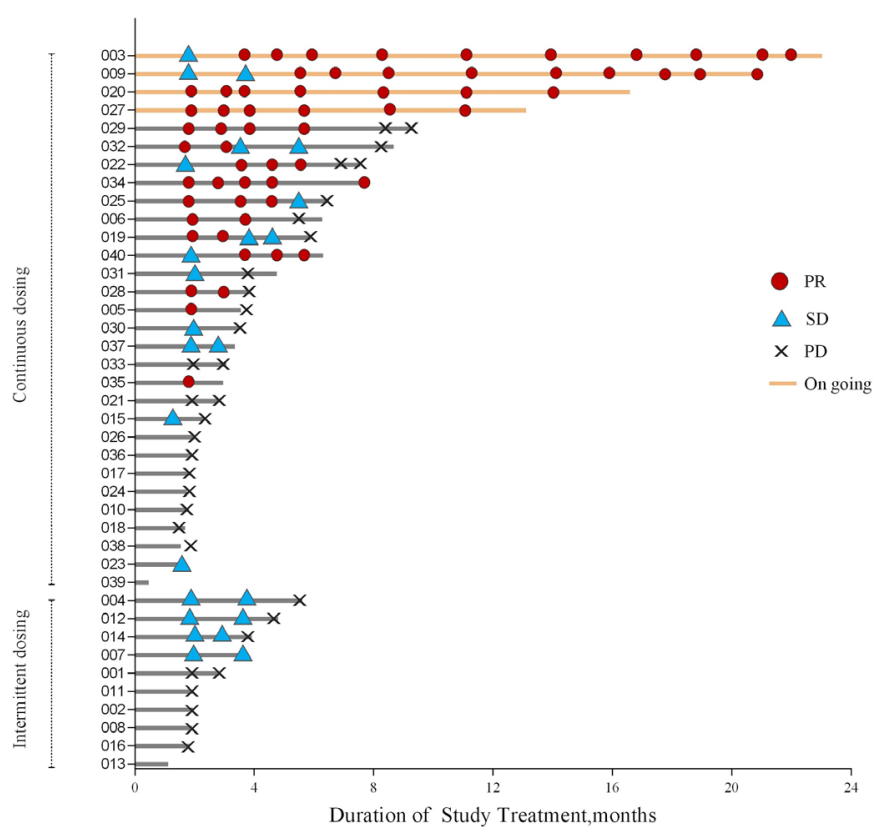

Figure 3 Duration of treatment and response. PD, progressive disease; PR, partial response; SD, stable disease.

carcinomas. ${ }^{14}$ By turning a 'cold' tumor into an 'inflamed' tumor with anti-VEGFR2 therapy, a tumor microenvironment more suitable for anti-PD-1 immunotherapy is created. Additionally, the small molecular VEGFR2 inhibitor apatinib is different from the macromolecular antiangiogenic agent bevacizumab, which in combination with chemotherapy has greatly improved PFS and ORR in HER2-negative metastatic breast cancer in many pivotal trials, but has a lack of OS improvement. ${ }^{25-28}$ Apatinib is an orally administered drug, which is more convenient for patients than bevacizumab; furthermore, besides tumor vasculature normalization effect, it also targets cancer stem-like cells, enhancing the efficacy of chemotherapy. ${ }^{29}$ The PFS and OS of patients who achieved response are significantly longer than the ones who did not respond to the combinational therapy, indicating the durability and long-lasting effects of immunotherapy. Together with the high ORR, this chemo-free combinational therapy is

A

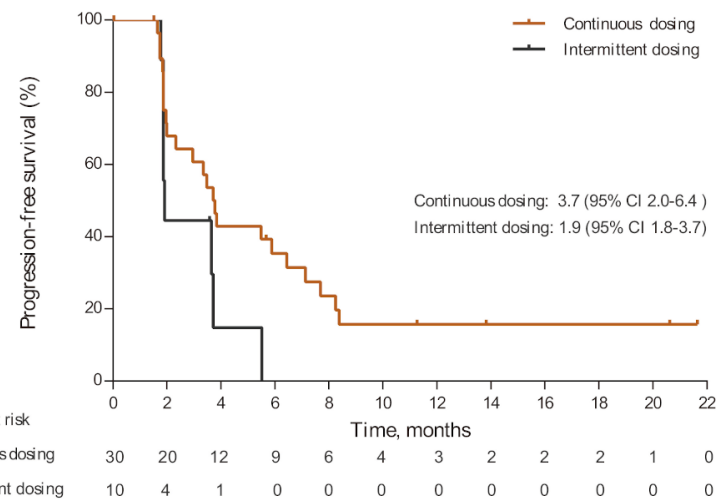

worthy of further large randomized controlled trials to validate its efficacy in advanced TNBC.

Hypertension, hand-foot syndrome, and proteinuria observed in this trial were associated with apatinib, whereas hypothyroidism, hyperthyroidism, pneumonitis, and capillary hemangioma were correlated with camrelizumab. Other AEs were probably be due to the combination therapy. The incidences of elevated AST/ alanine transaminase (ALT), hand-foot syndrome, and proteinuria in this trial were slightly higher than those observed in the apatinib $250 \mathrm{mg}$ plus camrelizumab cohort of a phase I trial of patients with advanced hepatocellular carcinoma and gastric cancer, while the rates of hypertension and hematological AEs were lower in our trial; other AEs observed in the current trial were at a similar incidence. ${ }^{20}$ The difference of AEs may be due to the different patient cohorts in this two trials. In addition to distinct solid tumor types between this two trials, our study only enrolled female patients, while the trial of advanced hepatocellular carcinoma and gastric cancer mainly enrolled male subjects $(94.4 \%) .{ }^{20}$ Overall, these AEs were relatively well tolerated, and the discontinuation and dose-reduction rates were both low. It is worth noting that this combinational chemo-free approach is highly effective in some patients that are resistant to several lines of chemotherapy.

Regarding the predictive biomarkers for the responses to combined therapy, we found that a high percentage of baseline stromal TILs was associated with more favorable outcomes. Previous studies also observed a positive association between stromal TILs and response to anti-PD-L1 monotherapy or PD-1 blockade plus trastuzumab. ${ }^{830}$

Although this is the first trial on anti-PD-1 and antiVEGFR2 combined therapy in advanced TNBC, it has several limitations. One restriction was the small sample size. A large randomized controlled trial is required to definitively define the role of such a combination therapy in TNBC. Moreover, this exploratory trial did not compare the efficacy of this apatinib-camrelizumab regimen with those of anti-PD-1/PD-L1 monotherapies or more recent combined chemoimmunotherapy. Further phase III randomized controlled trials are

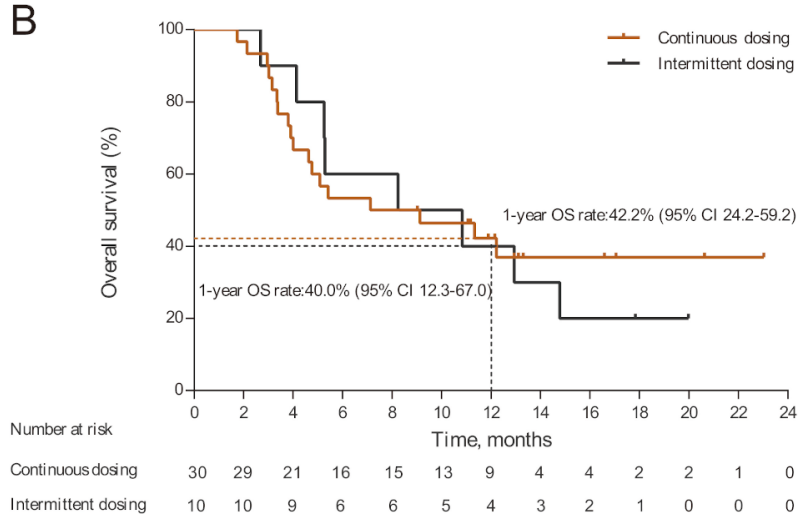

Figure 4 Kaplan-Meier curves for progression-free survival (A) and overall survival (OS) (B) in the apatinib continuous and the intermittent dosing cohorts. 
Table 3 Treatment-related adverse events

\begin{tabular}{|c|c|c|c|c|}
\hline & \multicolumn{2}{|c|}{ Continuous dosing cohort $(n=30)$} & \multicolumn{2}{|c|}{ Intermittent dosing cohort $(\mathrm{n}=10)$} \\
\hline & All grades & Grade 3 or 4 & All grades & Grade 3 or 4 \\
\hline All events & $30(100.0)$ & $8(26.7)$ & $9(90.0)$ & $2(20.0)$ \\
\hline Nausea & $4(13.3)$ & 0 & $2(20.0)$ & 0 \\
\hline Vomiting & $4(13.3)$ & 0 & $2(20.0)$ & 0 \\
\hline Fatigue & $14(46.7)$ & 0 & $9(90.0)$ & 0 \\
\hline Headache & $8(26.7)$ & 0 & $3(30.0)$ & 0 \\
\hline Hand-foot syndrome & $17(56.7)$ & $2(6.7)$ & $5(50.0)$ & 0 \\
\hline AST increased & $24(80.0)$ & $3(10.0)$ & $7(70.0)$ & $1(10.0)$ \\
\hline ALT increased & 19 (63.3) & 0 & $7(70.0)$ & $1(10.0)$ \\
\hline Blood bilirubin increased & $6(20.0)$ & 0 & $1(10.0)$ & 0 \\
\hline Diarrhea & $6(20.0)$ & 0 & $1(10.0)$ & 0 \\
\hline Hypertension & $11(36.7)$ & 0 & $3(30.0)$ & 0 \\
\hline Hematochezia & 0 & 0 & $1(10.0)$ & 0 \\
\hline Anemia & $5(16.7)$ & 0 & $2(20.0)$ & 0 \\
\hline Leukopenia & $9(30.0)$ & $1(3.3)$ & $3(30.0)$ & 0 \\
\hline Thrombocytopenia & $3(10.0)$ & 0 & $1(10.0)$ & 0 \\
\hline Proteinuria & 16 (53.3) & $1(3.3)$ & $5(50.0)$ & 0 \\
\hline Capillary hemangioma & $3(10.0)$ & 0 & $3(30.0)$ & 0 \\
\hline Rash $^{*}$ & $9(30)$ & 0 & $1(10.0)$ & 0 \\
\hline Gingival hemorrhage & $1(3.3)$ & 0 & $2(20.0)$ & 0 \\
\hline Cough & $3(10.0)$ & 0 & $1(10.0)$ & 0 \\
\hline Pneumonitis & $2(6.7)$ & $1(3.3)$ & 0 & 0 \\
\hline Hypothyroidism† & $8(26.7)$ & 0 & $1(10.0)$ & 0 \\
\hline Hyperthyroidism & $5(16.7)$ & 0 & 0 & 0 \\
\hline
\end{tabular}

Data are $\mathrm{n}(\%)$. There were no grade 5 adverse events in both arms.

*Includes rash, rash papular, rash pustular, dermatitis acneiform, and drug eruption.

†Includes hypothyroidism and increased concentrations of thyroid-stimulating hormone in blood.

AST, aspartate aminotransferase.

needed to directly compare apatinib in combination with camrelizumab versus camrelizumab monotherapy or with abraxane in treating advanced TNBC patients. Furthermore, although the ORR was high and the PFS was favorable in the apatinib continuous dosing cohort, the OS was still short in both cohorts. This might partially be due to nearly half of the enrolled patients refused any treatment after disease progression because of financial or other unspecified reasons $(14 / 30,46.7 \%)$.

In conclusion, camrelizumab combined with apatinib in treating advanced TNBC showed encouraging efficacy with a manageable safety profile, even in patients with PD-L1-negative tumors and those received one to two lines of chemotherapy. Future randomized controlled trials are needed to confirm these findings and to identify the subgroup of patients who can benefit from this combinational therapy.

\section{Author affiliations}

${ }^{1}$ Guangdong Provincial Key Laboratory of Malignant Tumor Epigenetics and Gene Regulation, Breast Tumor Center, Sun Yat-sen Memorial Hospital, Sun Yat-sen University, Guangzhou, China
${ }^{2}$ Jiangsu Hengrui Medicine Co., Ltd, Jiangsu, China

${ }^{3}$ Faculty of Medicine, Prince of Wales Hospital, The Chinese University of Hong Kong, Shatin, Hongkong, China

${ }^{4}$ Department of Oncology, The Fifth Medical Centre of Chinese PLA General Hospital, Beijing, China

${ }^{5}$ Fountain-Valley Institue for Life Sciences, 4th Floor, Building D, Guangzhou Institue of Biomedicine and Health, Chinese Academy of Sciences, Guangzhou, China

Acknowledgements We thank the patients and their families, nurses, trial coordinators, radiologists and pathologists who participated in this trial. Jiangsu Hengrui Medicine provided the study drug.

Contributors ES and QL: conceptualization, study design, project administration, and writing, review and editing. ZJ: conceptualization, and writing, review and editing. JL: methodology, data analysis, writing, original draft, and writing, review and editing. YL and QL: data collection and analysis. FS, HY, LJ, and YW: data collection and writing, review and editing. SS, QW, and WL: writing, review and editing. All authors read and approved the final manuscript.

Funding This work was supported by a grant from the Sun Yat-sen Memorial Hospital Clinical Research Program (SYS-Q-201701).

Competing interests None declared.

Patient consent for publication Not required. 
Ethics approval This study was conducted according to the Declaration of Helsinki and approved by the Institutional Review Board of Sun Yat-sen Memorial Hospital, and with a written informed consent obtained from all patients.

Provenance and peer review Not commissioned; externally peer reviewed.

Data availability statement Data are available on reasonable request. The datasets used and analyzed in the current study are available from the corresponding authors on reasonable request.

Open access This is an open access article distributed in accordance with the Creative Commons Attribution Non Commercial (CC BY-NC 4.0) license, which permits others to distribute, remix, adapt, build upon this work non-commercially, and license their derivative works on different terms, provided the original work is properly cited, appropriate credit is given, any changes made indicated, and the use is non-commercial. See http://creativecommons.org/licenses/by-nc/4.0/.

\section{ORCID iD}

Erwei Song http://orcid.org/0000-0002-5400-9049

\section{REFERENCES}

1 Li X, Yang J, Peng L, et al. Triple-negative breast cancer has worse overall survival and cause-specific survival than non-triple-negative breast cancer. Breast Cancer Res Treat 2017;161:279-87.

2 den Brok WD, Speers CH, Gondara L, et al. Survival with metastatic breast cancer based on initial presentation, de novo versus relapsed. Breast Cancer Res Treat 2017;161:549-56.

3 Gobbini E, Ezzalfani M, Dieras V, et al. Time trends of overall survival among metastatic breast cancer patients in the real-life ESME cohort. Eur J Cancer 2018;96:17-24.

4 Loi S, Sirtaine N, Piette F, et al. Prognostic and predictive value of tumor-infiltrating lymphocytes in a phase III randomized adjuvant breast cancer trial in node-positive breast cancer comparing the addition of docetaxel to doxorubicin with doxorubicin-based chemotherapy: big 02-98. J Clin Oncol 2013;31:860-7.

5 Adams S, Gray RJ, Demaria S, et al. Prognostic value of tumorinfiltrating lymphocytes in triple-negative breast cancers from two phase III randomized adjuvant breast cancer trials: ECOG 2197 and ECOG 1199. J Clin Oncol 2014;32:2959-66.

6 Loi S, Michiels S, Salgado R, et al. Tumor infiltrating lymphocytes are prognostic in triple negative breast cancer and predictive for trastuzumab benefit in early breast cancer: results from the FinHER trial. Ann Oncol 2014;25:1544-50.

7 Denkert C, von Minckwitz G, Brase JC, et al. Tumor-infiltrating lymphocytes and response to neoadjuvant chemotherapy with or without carboplatin in human epidermal growth factor receptor 2-positive and triple-negative primary breast cancers. J Clin Oncol 2015;33:983-91.

8 Emens LA, Cruz C, Eder JP, et al. Long-term clinical outcomes and biomarker analyses of Atezolizumab therapy for patients with metastatic triple-negative breast cancer: a phase 1 study. JAMA Oncol 2019;5:74-82.

9 Nanda R, Chow LQM, Dees EC, et al. Pembrolizumab in patients with advanced triple-negative breast cancer: phase Ib KEYNOTE-012 study. J Clin Oncol 2016;34:2460-7.

10 Dirix LY, Takacs I, Jerusalem G, et al. Avelumab, an anti-PD-L1 antibody, in patients with locally advanced or metastatic breast cancer: a phase 1B javelin solid tumor study. Breast Cancer Res Treat 2018;167:671-86.

11 Schmid P, Rugo HS, Adams S, et al. Atezolizumab plus nabpaclitaxel as first-line treatment for unresectable, locally advanced or metastatic triple-negative breast cancer (IMpassion130): updated efficacy results from a randomised, double-blind, placebo-controlled, phase 3 trial. Lancet Oncol 2020;21:44-59.

12 Schmittnaegel M, Rigamonti N, Kadioglu E, et al. Dual angiopoietin-2 and VEGFA inhibition elicits antitumor immunity that is enhanced by PD-1 checkpoint blockade. Sci Trans/ Med 2017;9:eaak9670.
13 Allen E, Jabouille A, Rivera LB, et al. Combined antiangiogenic and anti-PD-L1 therapy stimulates tumor immunity through HEV formation. Sci Transl Med 2017;9:eaak9679.

14 Li Q, Wang Y, Jia W, et al. Low-dose anti-angiogenic therapy sensitizes breast cancer to PD-1 blockade. Clin Cancer Res 2020;26:1712-24.

15 Scott AJ, Messersmith WA, Jimeno A. Apatinib: a promising oral antiangiogenic agent in the treatment of multiple solid tumors. Drugs Today 2015;51:223-9.

$16 \mathrm{Hu} \mathrm{X}$, Zhang J, Xu B, et al. Multicenter phase II study of apatinib, a novel VEGFR inhibitor in heavily pretreated patients with metastatic triple-negative breast cancer. Int J Cancer 2014;135:1961-9.

17 Fang W, Yang Y, Ma Y, et al. Camrelizumab (SHR-1210) alone or in combination with gemcitabine plus cisplatin for nasopharyngeal carcinoma: results from two single-arm, phase 1 trials. Lancet Oncol 2018;19:1338-50.

18 Mo H, Huang J, Xu J, et al. Safety, anti-tumour activity, and pharmacokinetics of fixed-dose SHR-1210, an anti-PD-1 antibody in advanced solid tumours: a dose-escalation, phase 1 study. $\mathrm{Br} \mathrm{J}$ Cancer 2018;119:538-45.

19 Huang J, Mo H, Zhang W, et al. Promising efficacy of SHR-1210, a novel anti-programmed cell death 1 antibody, in patients with advanced gastric and gastroesophageal junction cancer in China. Cancer 2019;125:742-9.

$20 \mathrm{Xu}$ J, Zhang Y, Jia R, et al. Anti-PD-1 antibody SHR-1210 combined with apatinib for advanced hepatocellular carcinoma, gastric, or esophagogastric junction cancer: an open-label, dose escalation and expansion study. Clin Cancer Res 2019;25:515-23.

21 Salgado R, Denkert C, Demaria S, et al. The evaluation of tumorinfiltrating lymphocytes (TILs) in breast cancer: recommendations by an international TILs Working group 2014. Ann Oncol 2015;26:259-71.

22 Simon R. Optimal two-stage designs for phase II clinical trials. Control Clin Trials 1989;10:1-10.

23 Tumeh PC, Harview CL, Yearley JH, et al. PD-1 blockade induces responses by inhibiting adaptive immune resistance. Nature 2014;515:568-71.

24 Rodriguez-Ruiz ME, Rodriguez I, Garasa S, et al. Abscopal effects of radiotherapy are enhanced by combined immunostimulatory mAbs and are dependent on CD8 T cells and Crosspriming. Cancer Res 2016;76:5994-6005.

25 Gray R, Bhattacharya S, Bowden C, et al. Independent review of E2100: a phase III trial of bevacizumab plus paclitaxel versus paclitaxel in women with metastatic breast cancer. J Clin Oncol 2009;27:4966-72.

26 Miles DW, Chan A, Dirix LY, et al. Phase III study of bevacizumab plus docetaxel compared with placebo plus docetaxel for the first-line treatment of human epidermal growth factor receptor 2-negative metastatic breast cancer. J Clin Oncol 2010;28:3239-47.

27 Robert NJ, Diéras V, Glaspy J, et al. RIBBON-1: randomized, doubleblind, placebo-controlled, phase III trial of chemotherapy with or without bevacizumab for first-line treatment of human epidermal growth factor receptor 2-negative, locally recurrent or metastatic breast cancer. J Clin Oncol 2011;29:1252-60.

28 Hey SP, Gyawali B, D'Andrea E, et al. A systematic review and meta-analysis of bevacizumab in first-line metastatic breast cancer: lessons for research and regulatory Enterprises. J Natl Cancer Inst 2020;112:335-42.

29 Tong X-zhen, Wang F, Liang S, et al. Apatinib (YN968D1) enhances the efficacy of conventional chemotherapeutical drugs in side population cells and ABCB1-overexpressing leukemia cells. Biochem Pharmacol 2012;83:586-97.

30 Loi S, Giobbie-Hurder A, Gombos A, et al. Pembrolizumab plus trastuzumab in trastuzumab-resistant, advanced, HER2-positive breast cancer (PANACEA): a single-arm, multicentre, phase $1 \mathrm{~b}-2$ trial. Lancet Oncol 2019;20:371-82. 